Mitteilungen der Österreichischen Geographischen Gesellschaft, 157. Jg. (Jahresband), Wien 2015, S. 363-369

\title{
Der Deutsche Kongress für Geographie 2015 in Berlin - ein kritischer Rückblick
}

\author{
Peter Alexander RumPolt, Wien/Innsbruck, und Marc Michael SEEBACHER, Wien* \\ mit 2 Abb. im Text
}

\section{Ein traditionsreicher Kongress mit neuem Namen}

Bei ihrer 59. Ausgabe wurde die für die deutschsprachige Geographie zentrale Tagung, der Deutsche Geographentag (DGT), erstmals als Deutscher Kongress für Geographie (DKG) bezeichnet. Der 59. „Geographentag“ und gleichsam quasi ,erste“ Deutsche Kongress für Geographie unter dieser Bezeichnung fand von 30. September bis 6. Oktober $2015^{1)}$ an der altehrwürdigen Humboldt-Universität zu Berlin statt. Zuletzt war der Geographentag im Jahr 1985 in Berlin, damals allerdings in West-Berlin, zu Gast gewesen.

Nach den Geographentagen 2007 in Bayreuth, 2009 in Wien (siehe auch MusiL 2009) sowie 2013 in Passau (siehe auch Rumpolt 2013a, 2013b) war der Deutsche Kongress für Geographie 2015 in Berlin der bereits vierte „Geographentag“ in Folge, welcher als ein Großkongress für Wissenschaft, Schule und Praxis und somit als gemeinsame Tagung für Hochschul-, Schul- und Berufsgeographen durchgeführt wurde. Mit dem neuen Namen der Tagung war nun auch der - bisher sprachlich rein maskuline - Personenbezug aus der Kongressbezeichnung entfernt worden.

\section{Tagungsort und 25 Jahre Deutsche Einheit}

Die Abhaltung des Deutschen Kongresses für Geographie 2015 in der deutschen Bundeshauptstadt Berlin gerade rund um den 3. Oktober 2015, den 25. Jahrestag der Wiedervereinigung der beiden deutschen Staaten im Jahr 1990, erwies sich als besondere raum-zeitliche Kongruenz. Morgens am Brandenburger Tor vorbei in Richtung Humboldt-Universität zu gehen und abends am Weg zum Hotel den Checkpoint Charlie in der Friedrichstraße zu passieren, wäre an sich schon etwas

\footnotetext{
* Mag. Peter Alexander Rumpolt, Bundesanstalt Statistik Österreich, Guglgasse 13, A-1110 Wien, und externer Projektmitarbeiter am Institut für Geographie, Universität Innsbruck, Innrain 52f, A-6020 Innsbruck; E-Mail: peter.rumpolt@statistik.gv.at, peter.rumpolt@univie.ac.at, http://www.statistik.at, http://www.uibk.ac.at/ geographie/; Univ.-Ass. Marc Michael SeEBAcher, BA MA, Institut für Geographie und Raumforschung, Karl-Franzens-Universität Graz, Heinrichstraße 36, A-8010 Graz; E-Mail: marc.seebacher@uni-graz.at, http://geographie.uni-graz.at

1) Formal wurde z.B. am Deck- und Titelblatt des Programmheftes, auf der Homepage sowie auf dem vor dem Hauptgebäude der Humboldt-Universität zu Berlin angebrachten Transparent (siehe Abb. 1) der Zeitraum 1.- 6. Oktober 2015 angegeben, de facto begann der Kongress aber bereits am 30. September. An diesem Tag wurden etliche Arbeitskreis- sowie Verbandssitzungen abgehalten.
} 
Besonderes gewesen. Das zeitliche Zusammentreffen der Tagung mit dem 25-jährigen Jubiläum der Deutschen Einheit machte einen Aufenthalt in Berlin zu dieser Zeit aber noch spezieller.

Des Jubiläums der Deutschen Einheit wurde am 3. Oktober 2015 in Berlin im Übrigen nicht nur am Brandenburger Tor gedacht, sondern beispielsweise auch im Berliner Olympiastadion: Vor Beginn eines Fußball-Bundesliga-Spieles wurden das Verschwinden der Berliner Mauer und das Zusammenwachsen von Ost- und West-Berlin anhand einer Fanchoreographie nachempfunden.

Als Standort der Tagung selbst war die „Unter den Linden“ gelegene Humboldt-Universität zu Berlin im Stadtteil Berlin-Mitte ausgewählt worden. Ebenso wie sechs Jahre zuvor beim Geographentag in Wien konnten somit beinahe alle Veranstaltungen im Rahmen des Kongresses räumlich konzentriert im Hauptgebäude der Universität sowie in einem weiteren, nahe gelegenen Universitätsgebäude abgehalten werden.

Die Möglichkeit zur Durchführung des Kongresses 2015 an einem solch zentralen und repräsentativen Ort in Berlin sei „,...] nicht zuletzt dem großen Engagement der Kolleginnen und Kollegen des Geographischen Instituts der Humboldt-Universität zu Berlin [zu verdanken] [...]“, so Werner Gamerith (2015, S. 7), Präsident der Deutschen Gesellschaft für Geographie (DGfG), in seinem Grußwort im Programmheft der Tagung. Für die Kongressorganisation und -koordination zeichneten Sylvana JAHRE und ihr Team verantwortlich, der Vorsitz des Ortsausschusses Berlin lag in den Händen des ehemaligen DGfG-Präsidenten Elmar KuLKE.

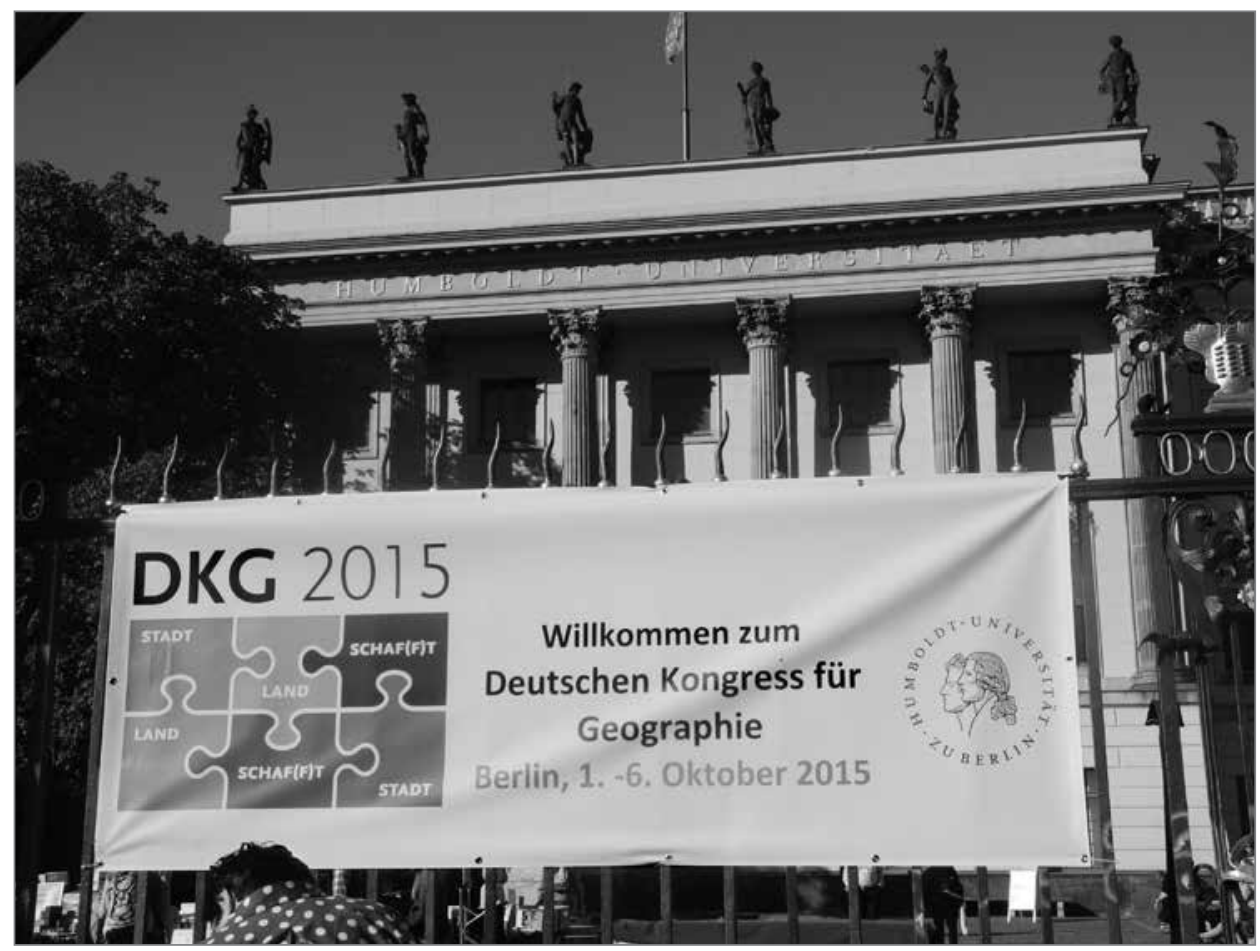

Foto: $\quad$ P.A. Rumpolt, 01.10 .2015

Abb. 1: Hinweis auf den Deutschen Kongress für Geographie 2015 vor dem Hauptgebäude der Humboldt-Universität zu Berlin (Berlin-Mitte, Unter den Linden 6) 


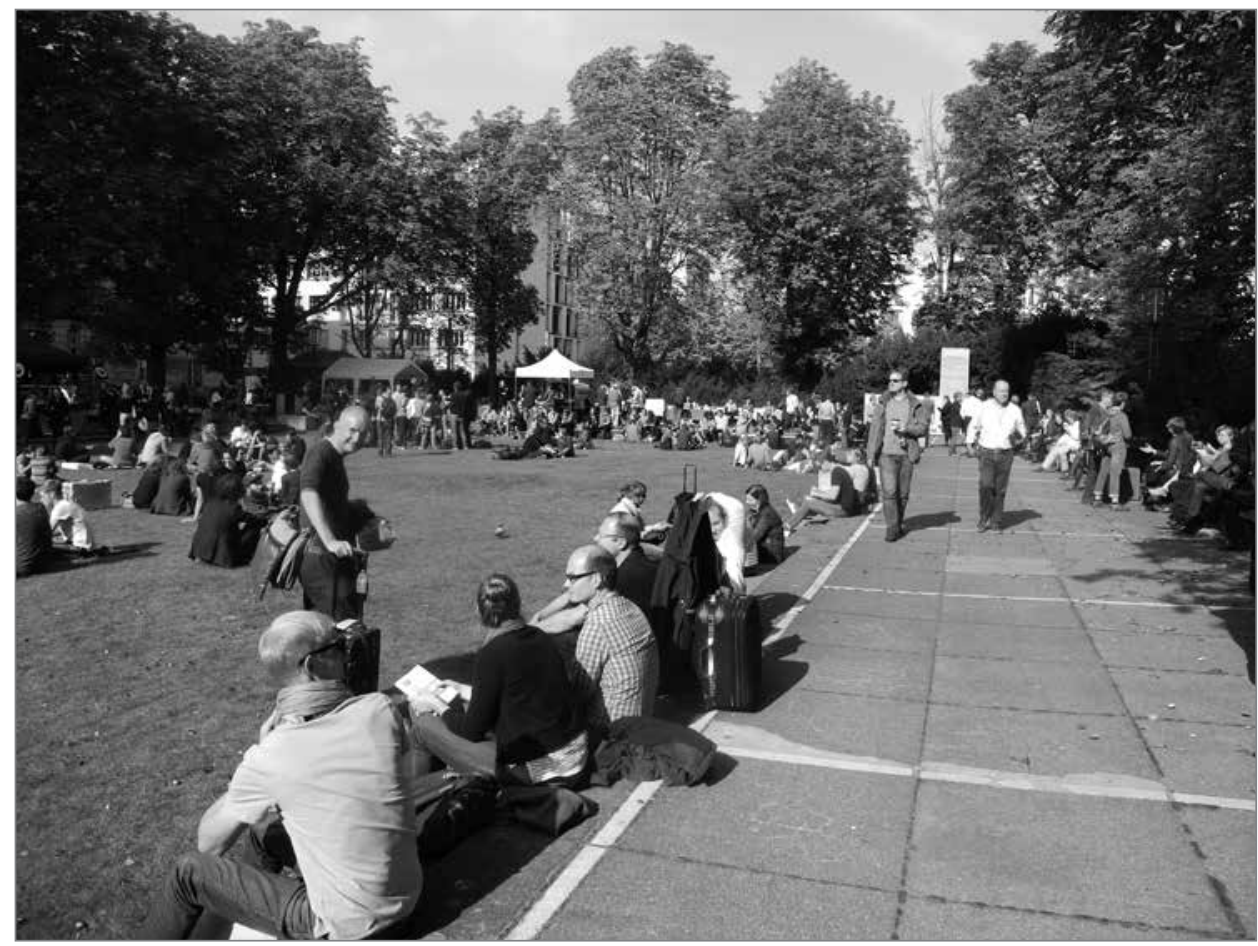

Foto: $\quad$ P.A. Rumpolt, 04.10.2015

Abb. 2: Während einer Kongresspause im Innenhof des Hauptgebäudes der Humboldt-Universität zu Berlin

\section{Tagungsmotto und Programmgestaltung}

Das Motto des „Geographentages“ 2015 war bereits 2013 in Passau bekanntgegeben worden; es lautete „Stadt Land Schaf(f)t - Land Schaf(f)t Stadt“"2). Dieses Thema weise auf rural-urbane und gesellschaftlich-naturräumliche Interdependenzen hin und könne den integrativen Charakter der wissenschaftlichen Disziplin der Geographie aufzeigen, welcher mit den Leitthemen und zugeordneten Fachsitzungen ausgeführt werde (vgl. KuLKe 2015, S. 8).

Denn obwohl sich die Strukturierung des Kongressprogrammes in seiner grundsätzlichen Ausrichtung an den bisherigen Traditionen der Veranstaltung orientierte, gab es diesbezüglich auch einige Veränderungen. Eine zentrale Neuerung betraf beispielsweise die Aufhebung der Differenzierung zwischen Leitthemensitzungen und Fachsitzungen. So wurde das Gros aller für die vier Kerntage des Kongresses in Berlin (1. bis 4. Oktober 2015) angekündigten Sessions von 240 Fachsitzungen gebildet, deren Ausgestaltung auch hinsichtlich der Wahl des Formats der jeweiligen Sitzungslei-

2) Eine ähnliche bewusste sprachliche Doppeldeutigkeit wird seit September 2013 auch im Titel der Regionalzeitschrift des Biosphärenparks Großes Walsertal in Vorarlberg verwendet: Bei der Zeitschrift „talschafft“ wird das zweite $\mathrm{f}$ zwar nicht in Klammern, jedoch in einer anderen Schriftfarbe visualisiert. 
tung überlassen wurde. Die Fachsitzungen waren den folgenden acht Leitthemen zugeordnet (vgl. OrTSAusschuss BerLin 2015, S. 27):

- Stadt und Land: Kontinuitäten und Konflikte

- Vernetzung und Entwicklungspfade

- Demographie im 21. Jahrhundert

- Umwelt und Gerechtigkeit

- Landnutzungswandel und Nachhaltigkeit

- Erde: Lebensraum und Risiko

- Didaktik der Geographie

- Theoretische, methodische und disziplinäre Reflexion

Konkretisiert wurde ihre inhaltliche Ausrichtung im Programmheft durch das exemplarische Auflisten schlagwortartiger Unterpunkte (siehe ebd.). Die Leitthemen, so kann dem Programmheft entnommen werden, seien dabei explizit nicht als Abbildung der Teildisziplinen des Faches oder einer Differenzierung zwischen Schule, Wissenschaft und Praxis gedacht, sondern als themenorientierte, ,entgrenzte“ Querschnittsfelder. Sie könnten den innerfachlichen Austausch, die Vernetzung sowie auch das Potenzial geographischer Forschung für die Gesellschaft betonen.

Eine weitere interessante Neuerung im Rahmen des Kongresses stellte die Aufnahme von sogenannten Journal Lectures in das Programm dar. Bei diesen Veranstaltungen standen unter dem organisatorischen ,Dach` einer renommierten geographischen Fachzeitschrift (z.B. Geographische Zeitschrift, Die Erde, Geographica Helvetica, Raumforschung und Raumordnung oder Political Geography) jeweils themenspezifische Vorträge internationaler Fachkollegen und/oder unterschiedliche Diskussionsformate im Mittelpunkt.

Neben diesen Neuerungen wurden aber auch ,klassische Elemente' als Teil des Programmes fortgeführt. So gab es auch beim diesjährigen Kongress in Berlin eine Reihe von Keynote-Vorträgen - mit insgesamt zehn sogar deutlich mehr als beispielsweise 2013 in Passau -, welche gleicherweise human- und physiogeographische wie auch geographiedidaktische Themen- und Fragestellungen ansprachen. Besonders herauszustreichen ist der hohe Aktualitätsbezug der humangeographischen Keynotes, welche sich aus unterschiedlichen Blickwinkeln mit der komplexen Thematik von Flucht, Asyl und Migration auseinandersetzten und dabei z.B. Fragen nach den gegenwärtigen Funktionen von Grenzen, nach der Formierung von nationalen Identitäten sowie auch nach der sozialen Topographie von Vorurteilen anhand von empirischen Beispielen aus dem europäischen Kontext intensiv diskutierten. Somit wurde hier die gesellschaftspolitische Relevanz einer geographischen Betrachtungsperspektive oder die Notwendigkeit, aktuelle sozialräumliche Prozesse und Phänomene von einer theoretisch-konzeptionell informierten Position aus kritisch zu reflektieren, klar aufgezeigt.

Weitere - auch bereits von den vergangenen „Geographentagen“ her bekannte - Eckpfeiler des Kongresses in Berlin bildeten Posterpräsentationen, die entsprechend den Leitthemen der Veranstaltung strukturiert waren, eine Fach- und Verlagsausstellung, ein umfangreiches Exkursionsprogramm, das sowohl auf die Stadt Berlin als auch auf das Umland ausgerichtet und in Schwerpunkte differenziert war (Physische Geographie, Humangeographie, Fachdidaktik, Studentische Exkursionen), sowie Arbeitskreis- und Verbandssitzungen. Ausgehend von Studenten des Geographischen Instituts der Humboldt-Universität zu Berlin wurde auch ein „Junger Kongress für Geographie“(JKG) mit eigenen Sitzungen und Vorträgen sowie Diskussionsmöglichkeiten abgehalten.

In den Kongress eingebunden waren auch ein vom Verband Deutscher Schulgeographen (VDSG) organisierter, zweitägiger „Tag der Schulgeographie“ am 2. und 3. Oktober, ein vom Deutschen Verband für Angewandte Geographie (DVAG) veranstalteter „Tag der Angewandten Geographie“ am 
3. Oktober sowie die Jahrestagung des Arbeitskreises Geomorphologie am 3. und 4. Oktober 2015. Der Tag der Schulgeographie stand unter dem Leitthema „Unsere Erde: Lebensraum und Risiko“. Beim Tag der Angewandten Geographie wurde auch auf 25 Jahre Deutsche Einheit explizit Bezug genommen, wobei dieses Jubiläum mit einem speziellen Fokus auf Prozesse der Raumentwicklung und Fragen nach (räumlicher) Gleichheit und (raumbezogener) Gerechtigkeit näher beleuchtet wurde. Weiters wurde das inhaltliche Programm des Kongresses für Geographie im Jahr 2015 auch noch durch eine Reihe von Sonderveranstaltungen ergänzt: So fand beispielsweise am Abend des 3 . Oktober eine hochkarätig besetzte Podiumsdiskussion zum Verhältnis von Geographie und Gesellschaftstheorie statt, bei welcher auch die „österreichische Geographie“ vertreten war.

Von der in Passau 2013 eingeführten zentralen Festveranstaltung inklusive eines geselligen Teiles wurde diesmal wieder Abstand genommen. Vielmehr bildeten heuer eine Eröffnungsveranstaltung im Audimax der Humboldt-Universität zu Berlin am Abend des 1. Oktober, bei welcher neben den musikalisch umrahmten Eröffnungsansprachen vor allem die feierliche Verleihung von wissenschaftlichen Preisen im Mittelpunkt stand, sowie ein „Get-together“ in lockerer Atmosphäre in den Räumlichkeiten der Mensa Nord (am Gelände der Charité) am Abend des 2. Oktober das Rahmenprogramm des Kongresses. Aufgrund einer Sportverletzung konnte der amtierende Präsident der DGfG, Werner GAMERITH (Universität Passau), bedauerlicherweise nicht an diesen Programmpunkten teilnehmen und musste sich von Ingrid HEMMER (Universität Eichstätt-Ingolstadt) vertreten lassen.

\section{4 (Kritische) Reflexionen}

Der Deutsche Kongress für Geographie in Berlin kann im Rückblick als eine gelungene wissenschaftliche Großveranstaltung bewertet werden. Der Tagungsort an der prominenten Adresse „Unter den Linden“, mitten im urbanen Zentrum der deutschen Hauptstadt, war der größten wissenschaftlichen Veranstaltung der deutschsprachigen Geographie wahrlich würdig. Das schöne Herbstwetter hat sich positiv auf die kollegialen Gespräche in dem mit vielen Ständen zur Verköstigung mit Speisen aus unterschiedlichen Kulturkreisen ausgestatteten Innenhof (siehe Abb. 2) des altehrwürdigen Universitätsgebäudes ausgewirkt, und auch die Organisation schien - aus der Außenperspektive der Verfasser beurteilt - gut zu funktionieren. Das Angebot, in den Pausen zwischen den Sessions kostenlos Fruchtsäfte, Kaffee, Kekse u.a. konsumieren zu können, sei zur Nachahmung empfohlen - ebenso die zweifellos der Müllvermeidung dienende Möglichkeit zum Erwerb eines speziellen Porzellanbechers für den Kaffeekonsum während des Kongresses.

Eine detaillierte und umfassende (inhaltliche) Diskussion aller Teilveranstaltungen kann an dieser Stelle, allein schon aufgrund deren großer Zahl und Heterogenität, nicht vorgenommen werden. Wie aber bereits im Rückblick auf den Geographentag 2013 in Passau angemerkt (vgl. RuMPOLT 2013a, S. 326) war durchaus auch in Berlin eine Unterrepräsentanz der Physischen Geographie in den Fachsitzungen zu beobachten. Die Strukturierung des Programmes anhand der - integrativen - Leitthemen brachte es jedoch mit sich, dass nicht so klar zwischen Humangeographie und Physischer Geographie zu unterscheiden war. Die Zuteilung einzelner Fachsitzungen zu Leitthemen, deren thematische Abgrenzungen selbst vielfach fließend waren, erschien oft willkürlich - was aber, wegen der großen inhaltlichen Breite der Tagungsbeiträge wohl auch nicht leicht zu verhindern war. Manche Fachsitzungen, so die äußerst interessante Vorstellung einer Studie zum Image der Geographie in Deutschland, hätten eventuell als Sonderveranstaltungen ausgewiesen werden können, um sie noch , sichtbarer' zu machen.

Auch das Programmheft hätte übersichtlicher sein können. War nämlich seine graphische Gestaltung äußerst ansprechend, so erschwerten die acht für die Leitthemen gewählten und sehr ähn- 
lichen Farbtöne sowie die ,fließenden` Übergänge zwischen den einzelnen Kongresstagen doch die rasche Orientierung. Der Umfang des Programmhefts (eigentlich ,-buches') wirft doch auch die Frage auf, ob die Fülle des Programms (und damit auch die Größe der Tagung) überhaupt noch ein sinnvolles Maß hat.

Als praktisches Problem hat sich das individuelle Wechseln zwischen verschiedenen Sitzungen erwiesen. Während dies bei früheren Geographentagen infolge einer einheitlich vorgegebenen zeitlichen Struktur der Sitzungen gut möglich war, wurde es diesmal deutlich erschwert: vor allem durch die Individualisierung des Ablaufs von Sitzungen in Bezug auf Anzahl und Länge der Vorträge sowie die Integration von Diskussionsmöglichkeiten; aber auch durch das Fehlen von Übersichtslisten zu den jeweils in einem Raum stattfindenden Sitzungen im jeweiligen Eingangsbereich. Auch fielen etliche Veranstaltungen aus, womit sich offenbar eine bereits in Passau feststellbare Tendenz fortsetzte.

Positiv zu bewerten ist ein Trend zur Internationalisierung. So sprachen die prominenten Keynote-Speaker nicht nur aktuelle, politisch relevante Themenfelder an, sondern sorgten auch für einen begrüßenswerten Austausch zwischen der deutschsprachigen Geographie und der internationalen scientific community. Dies ist ein auch im Hinblick auf zukünftige Geographiekongresse wichtiger und wohl zu verstärkender Aspekt. Allerdings muss auch der Unterschied zwischen ,regionalen“ Veranstaltungen (wie dieser des deutschen Sprachraums), die sich auch an Schul- und Berufsgeographen richten, und globalen Kongressen wie jenen der Internationalen Geographischen Union (IGU) gewahrt werden.

\section{Ausblick}

Wenngleich dieses geographische Großereignis also als sehr gut organisiert, erfolgreich durchgeführt und durch innovative Ideen charakterisiert bezeichnet werden kann, so könnten manche der oben dargelegten Beobachtungen doch zum Nachdenken anregen.

Allzu viel Zeit bleibt dafür allerdings nicht, denn die nächste Ausgabe des „Geographentages“ ist bereits in Planung. Ihr Austragungsort wird im Jahr 2017 die baden-württembergische Universitätsstadt Tübingen sein. Der Forschungsbereich Geographie der renommierten Eberhard-Karls-Universität Tübingen zeichnet für Organisation und Durchführung verantwortlich. Tübingen wird als kleine Stadt nach der Millionenmetropole Berlin sicherlich einen reizvollen Kontrast bilden.

\section{Literaturverzeichnis}

Gamerith W. (2015), Grußwort der Deutschen Gesellschaft für Geographie (DGfG). In: OrTSAusSCHUSS BerLin (Hrsg.), Deutscher Kongress für Geographie. 1.-6. Oktober 2015, S. 7. Berlin.

KulKe E. (2015), Grußwort des Ortsausschusses Berlin. In: Ortsausschuss Berlin (Hrsg.), Deutscher Kongress für Geographie. 1.-6. Oktober 2015, S. 8. Berlin.

Musil R. (2009), Der Deutsche Geographentag in Wien 2009 - eine wissenschaftliche Großveranstaltung. In: Mitteilungen der Österreichischen Geographischen Gesellschaft, 151, S. 315-322.

Ortsausschuss BerLin [Humboldt-Universität zu Berlin. Ortsausschuss des Deutschen Kongresses für Geographie 2015 im Auftrag der Deutschen Gesellschaft für Geographie (DGfG). Geographisches Institut] (Hrsg.) (2015), Deutscher Kongress für Geographie. 1.-6. Oktober 2015. Berlin. Programm. 
Rumpolt P.A. (2013a), Der 58. Deutsche Geographentag 2013 in Passau - ein großer Kongress in einer kleinen Stadt. In: Mitteilungen der Österreichischen Geographischen Gesellschaft, 155, S. 321-326.

Rumpolt P.A. (2013b), Geographentag 2013 in Passau - ein Rückblick. In: Geographie aktuell. Informationen der Österreichischen Geographischen Gesellschaft, 5, 4, S. 3.; auch online unter: http://www.geoaustria.ac.at/items/uploads/files/ga2013_4_online(1).pdf(23.11.2015).

Rumpolt P.A., Seebacher M.M. (2015), Deutscher Kongress für Geographie 2015 in Berlin - ein Rückblick. In: Geographie aktuell. Informationen der Österreichischen Geographischen Gesellschaft, 7, 4, S. 5. (im Druck) 